\title{
Phase diagram and critical behavior of the pair annihilation model
}

\author{
Adriana Gomes Dickman ${ }^{1, *}$ and Ronald Dickman ${ }^{2, \dagger}$ \\ ${ }^{1}$ Departamento de Física e Química, Pontifícia Universidade Católica de Minas \\ Gerais, Av. Dom José Gaspar, 500, Coração Eucarístico, 30535-901, Belo Horizonte, Minas \\ Gerais, Brazil \\ ${ }^{2}$ Departamento de Física, ICEx, and Universidade Federal de Minas Gerais \\ Caixa Postal 702, 30123-970, Belo Horizonte, Minas Gerais, Brazil, \\ National Institute of Science and Technology for Complex Systems, \\ Caixa Postal 702, 30161-970 Belo Horizonte, Minas Gerais, Brazil
}

We study the critical behavior of the pair annihilation model (PAM) with diffusion in one, two and three dimensions, using the pair approximation (PA) and Monte Carlo simulation. Of principal interest is the dependence of the critical creation rate, $\lambda_{c}$, on the diffusion probability $D$, in particular, whether survival is possible at arbitrarily small creation rates, for sufficiently rapid diffusion. Whilst the PA predicts that in any spatial dimension $d \geq 1$, $\lambda_{c} \rightarrow 0$ at some diffusion probability $D^{*}<1$, Katori and Konno [Physica A 186, 578 (1992)] showed rigorously that for $d \leq 2$, one has $\lambda_{c}>0$ for any $D<1$. Our simulation results are consistent with this theorem. In two dimensions, the extinction region becomes narrow as $D$ approaches unity, following $\lambda_{c} \propto \exp \left[-\right.$ const. $\left./(1-\mathrm{D})^{\gamma}\right]$, with $\gamma=1.41(2)$. In three dimensions we find $D^{*}=0.333(3)$. The simulation results confirm that the PAM belongs to the directed percolation universality class.

PACS numbers: 02.50.Ey, 05.70.Ln, 05.50.+q

electronic address: *adickman@pucminas.br 
electronic address: ${ }^{\dagger}$ dickman@fisica.ufmg.br 


\section{INTRODUCTION}

In recent decades, general theories of phase transitions and critical phenomena have been developed, unifying our understanding of equilibrium phase transitions in liquid-vapor, magnetic, liquid crystals and other systems. By contrast, the study of nonequilibrium critical phenomena is still in development. Since the transition rates in such systems do not satisfy detailed balance, the steady-state probability distribution in these systems is not known a priori, and the analysis must be based upon the dynamics. Starting from the basic contact process [1], many particle systems have been studied in efforts to characterize scaling properties at nonequilibrium phase transitions [2 4]. These models, which involve creation and annihilation of particles on a lattice, typically exhibit a phase transition to an absorbing state (one allowing no escape), and so violate the detailed balance principle. An issue that has attracted some interest is the combined effect of multiparticle rules and diffusion (hopping), which tends to spread particles uniformly over the system.

In this work we revisit the pair annihilation model (PAM) [5, 6]. In this model particles diffuse on a lattice at rate $D$, nearest-neighbor pairs of particles are annihilated at rate $(1-D) /(1+\lambda)$, and particles attempt to create new particles at rate $(1-D) \lambda /(1+\lambda)$. Double occupancy of sites is forbidden. The model exhibits active and absorbing phases, separated by a continuous phase transition at $\lambda_{c}(D)$. Using cluster approximations and Monte Carlo simulation, we determine the phase boundary in one, two, and three dimensions.

The pair approximation predicts that for a diffusion rate greater than a certain value, $D^{*}$, the critical parameter $\lambda_{c}=0$. (That is, for $D>D^{*}$, an arbitrarily small creation rate is sufficient to maintain a nonzero particle density.) This prediction is known to be wrong in dimensions $d \leq 2$ : Katori and Konno [7] proved that $\lambda_{c}>0$ for any diffusion probability $D<1$, in one and two dimensions. Their theorem is based on a relation between the PAM and the branching annihilating random walk of Bramson and Gray [8]. Existence of a $D^{*}<1$ is not ruled out in $d \geq 3$ dimensions. The difference is connected with the nonrecurrence of random walks in $d \geq 3[7]$. How $\lambda_{c}$ tends to zero as $D \rightarrow 1$ is, however, unknown. Moreover the question of whether, in three or more dimensions, $D^{*}$ is in fact less that unity, has not, to our knowledge, been studied. The principal motivation for the present work is to determine $\lambda_{c}(D)$ via numerical simulation. We also verify that the model belongs to the directed percolation (DP) universality class, as expected on the basis of symmetry considerations 
[9, 10]. Our simulation results, while consistent with the Katori-Konno theorem, show that in the two-dimensional case, $\lambda_{c}$ becomes extremely small as $D$ approaches unity, possibly suggesting the (incorrect) impression that the critical value is actually zero at some finite diffusion rate.

The remainder of this paper is organized as follows. In the following section (II) we define the model and discuss its limiting behaviors in the $\lambda-D$ plane. Then in Sec. III we present, for completeness, the one- and two-site cluster approximations. Simulation results are discussed in Sec. IV, followed by a brief discussion in Sec. V.

\section{THE MODEL}

The PAM is defined on a lattice, in which sites can be either occupied by a particle or vacant [2, 5, 6]; we denote these states by $\sigma_{x}=1$ (site $x$ occupied) and $\sigma_{x}=0$ (site $x$ vacant). There are three kinds of transition: nearest-neighbor (NN) hopping ("diffusion"), creation, and pairwise annihilation, with associated rates $D,(1-D) \lambda /(1+\lambda)$, and $(1-D) /(1+\lambda)$, respectively. (Since the rates are parameterized so as to sum to unity, we are free to refer to $D$ as the diffusion probability.) At each step of the evolution, the next attempted transition is taken as diffusion, creation, or annihilation, with probabilities $D,(1-D) \lambda /(1+\lambda)$, and $(1-D) /(1+\lambda)$, respectively.

In a hopping transition, a site $x$ is chosen at random, along with a nearest-neighbor site $y$ of $x$. Then if $\sigma_{x} \neq \sigma_{y}$, the states are exchanged. In a creation event, a site $x$ is chosen. If $\sigma_{x}=1$, a nearest-neighbor $y$ is chosen, and if $\sigma_{y}=0$ this variable is set to one. Finally, in an annihilation event, a pair of nearest-neighbor sites $x$ and $y$ are chosen at random, and if $\sigma_{x}=\sigma_{y}=1$, both variables are set to zero. Each transition corresponds to a time increment $\Delta t=1 / N_{\text {site }}$, where $N_{\text {site }}$ is the number of lattice sites.

To improve efficiency, in simulations the site $x$ is chosen from a list of occupied sites; then the time increment is $\Delta t=1 / N_{p}$, with $N_{p}$ the number of particles in the system, immediately prior to the transition. In this implementation, the rate of annihilation of a given NN particle pair is

$$
R_{a n}=\frac{1}{\Delta t} \frac{1-D}{1+\lambda} \frac{2}{N_{p}} \frac{1}{2 d}=\frac{1}{d} \frac{1-D}{1+\lambda}
$$

where the factor $2 / N_{p}$ arises because either particle in the pair can be selected from the list 
of $N_{p}$ particles.

The particle-free configuration is absorbing. By analogy with the contact process [1, 2], we expect that in the infinite-size limit the system undergoes a phase transition between an active state (with nonzero stationary particle density) and an absorbing one, as one crosses the critical line $\lambda_{c}(D)$ in the $\lambda$-D plane. As creation depends upon a single particle, the order parameter is the stationary density of particles, $\rho$.

When a new particle is created, it always forms a pair with the "parent" particle, making these two particles susceptible to annihilation. In the active stationary state, increasing $D$ at fixed $\lambda$ tends to reduce the fraction of nearest-neighbor particle pairs toward its random mixing value, $\rho^{2}$. Thus we should expect $\lambda_{c}$ to be a decreasing (or at least, nonincreasing) function of $D$. In the simplest mean-field theory analysis, the annihilation rate is proportional to $\rho^{2}$, so that for small $\rho$, one has $\dot{\rho} \propto \lambda \rho$ - const. $\times \rho^{2}$, which admits a stationary solution $\rho \propto \lambda$ for any nonzero creation rate. In the limit $D \rightarrow 1$ we expect mean-field theory to hold, so that $\lambda_{c} \rightarrow 0$ in this limit. This raises the question of whether $\lambda_{c}$ vanishes at some diffusion probability $D^{*}$ strictly less than unity. While the two-site approximation predicts $D^{*}<1$ in any dimension, the results of Katori and Konno [7] imply that $D^{*}=1$ in dimensions $d \leq 2$.

The phase diagram of the PAM is expected to have the form shown in Fig. 1. For $D<D^{*}$ the behavior along the critical line $\lambda_{c}(D)$ should be that of DP, given that such behavior is generic for absorbing-state phase transitions without special symmetries or conserved quantities [9, 10]. If $D^{*}<1$, then we expect mean-field like critical behavior as $\lambda \rightarrow \lambda_{c}=0$ at fixed $D>D^{*}$. Within the absorbing phase, for $0<\lambda<\lambda_{c}(D)$, an isolated particle can produce an offspring, leading to annihilation of both the original and the new particle. On the line $\lambda=0$, this channel to annihilation is not available, and isolated particles cannot disappear. Thus the dynamics at long times, for $D>0$, will be that of the diffusive annihilation process $A+A \rightarrow 0$, for which the particle density $\rho(t)$ decays $\sim 1 / \sqrt{t}$ in $d=1$, $\sim(\ln t) / t$ in two dimensions, and $\sim 1 / t$ in $d \geq 3[11,12]$. Finally, at the point $\lambda=D=0$, starting from all sites occupied, pairs are annihilated successively until only isolated particles remain. This is equivalent to the random sequential adsorption (RSA) of dimers. (In the present case, of course, dimers are removed not adsorbed, so the final particle density is $1-2 \theta_{\infty}$, where $\theta_{\infty}$ is the final coverage in RSA.) On the line, the final density of isolated particles is $e^{-2}=0.135335 \ldots$ [13], while in two dimensions one has $\rho_{\infty} \simeq 0.093108$ (8) [14]. 
One may anticipate interesting crossover behaviors in the vicinity of one or another limit. In the present work, however, we focus on determining the function $\lambda_{c}(D)$ using Monte Carlo simulation.

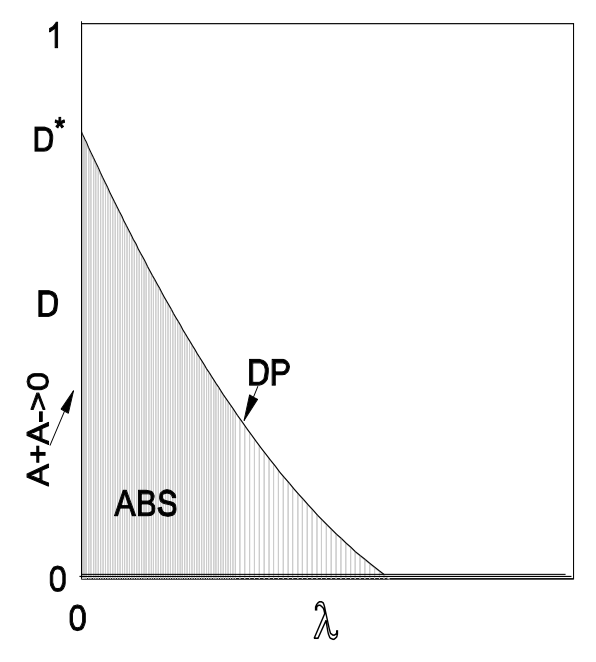

FIG. 1: Schematic phase diagram of the PAM in the $\lambda-D$ plane. The results of [7] imply that $D^{*}=1$ in one and two dimensions.

\section{CLUSTER APPROXIMATIONS}

In this section we study the PAM through mean-field - site and pair approximations [15]. In general, mean-field results provide a good qualitative description of the phase diagram and give an order-of-magnitude estimate of the critical point. $n$-site approximations are a natural way to improve the mean-field approach. The method consists of treating the transitions inside clusters of $n$ sites exactly, while transitions involving sites outside the cluster are treated in an approximate manner. 


\section{A. One-site approximation}

Let $\rho=\operatorname{Prob}\left(\sigma_{x}=1\right)$ denote the density of particles. The density is governed by,

$$
\begin{aligned}
\frac{d \rho}{d t} & =\frac{1}{2 d}(1-D) \frac{\lambda}{1+\lambda} \sum_{\hat{e}} P\left(\sigma_{x}=0, \sigma_{x+\hat{e}}=1\right) \\
& -\frac{1}{d} \frac{1-D}{1+\lambda} \sum_{\hat{e}} P\left(\sigma_{x}=1, \sigma_{x+\hat{e}}=1\right) \\
& -D \sum_{\hat{e}} P\left(\sigma_{x}=1, \sigma_{x+\hat{e}}=0\right) \\
& +D \sum_{\hat{e}} P\left(\sigma_{x}=0, \sigma_{x+\hat{e}}=1\right),
\end{aligned}
$$

where the sums are over the $2 d$ nearest-neighbors of site $x$, and $P\left(\sigma_{x}, \sigma_{x+\hat{e}}\right)$ is a two-site joint probability. Equation (2) couples the one-site probability $\rho$ to the two-site probabilities, which in turn depend on the three-site probabilities, and so forth, leading to an infinite hierarchy of equations for the $n$-site probabilities. The site-approximation consists in truncating this hierarchy at $n=1$, so that the two-site probabilities are replaced by a product of two one-site probabilities. Assuming spatial homogeneity and isotropy we obtain the following equation for $\rho$,

$$
\frac{d \rho}{d t}=\frac{1-D}{1+\lambda}\left[\lambda \rho-(2+\lambda) \rho^{2}\right]
$$

The stationary solutions are $\bar{\rho}=0$ (unstable for $\lambda>0$ ) and $\bar{\rho}=\lambda /(2+\lambda)$. Thus, in this approximation the critical parameter $\lambda_{c}$ is zero in any dimension. Notice that in this approximation the diffusion rate has no influence on the stationary solution.

\section{B. Pair approximation}

To derive the pair approximation equations, we consider the changes in the configuration of a NN pair of sites (the central pair), given the states of the surrounding sites. Using the symbols $\circ$ and $\bullet$ to represent, respectively, vacant and occupied sites, the states of a pair are $\diamond, \bullet \bullet, \bullet \circ$, and $\bullet \bullet$; the latter two have the same probability and may be treated as a single class using appropriate symmetry factors. It is convenient to use $(\bullet \bullet)$ to denote the fraction of $\bullet \bullet$ pairs, and so on. Then we have for the site fractions $(\bullet)=\rho$ and $(\circ)=1-\rho$ : 


$$
\begin{aligned}
& (\bullet)=(\bullet \bullet)+(\bullet \circ), \\
& (\circ)=(\circ \circ)+(\bullet \circ) .
\end{aligned}
$$

The pair fractions satisfy $(\circ \circ)+2(\circ \bullet)+(\bullet \bullet)=1$. The pair approximation consists in writing the joint probability of a set of three neighboring sites in the form $(a b c)=(a b)(b c) /(b)$.

There are five possible transitions between the pair states. Consider for example the transition $\circ \circ \rightarrow \circ \bullet$. This can occur via creation or via hopping, if and only if the rightmost site of the central pair has an occupied NN. Since its NN within the central pair is vacant, at least one of its $2 d-1 \mathrm{NNs}$ outside the central pair must be occupied. The rate of transitions via creation is

$$
R_{1, c}=(1-D) \tilde{\lambda} \frac{2 d-1}{2 d} \frac{(\circ \circ)(\circ \bullet)}{(\circ)}
$$

where we introduced $\tilde{\lambda}=\lambda /(1+\lambda)$. Adding the contribution due to diffusion, we obtain the total rate for this transition,

$$
R_{1}=\frac{2 d-1}{2 d} \frac{(\circ \circ)(\circ \bullet)}{(\circ)}[D+(1-D) \tilde{\lambda}]
$$

Note that the contribution to the loss term for (०o) associated with this process is $2 R_{1}$, due to the mirror transition $\diamond \circ \rightarrow \bullet \circ$.

The rates for the other transitions are:

$\infty \bullet \rightarrow \circ:$

$$
\begin{gathered}
R_{2}=\frac{2 d-1}{2 d} \frac{(\circ \bullet)}{(\bullet)}[D(\circ \bullet)+2(1-D)(1-\tilde{\lambda})(\bullet \bullet)] \\
R_{3}=\frac{2 d-1}{2 d} \frac{(\circ \bullet)^{2}}{(\circ)}[D+(1-D) \tilde{\lambda}]+\frac{1}{2 d}(1-D) \tilde{\lambda}(\circ \bullet)
\end{gathered}
$$

$\bullet \bullet \rightarrow \circ:$

$$
R_{4}=\frac{1}{d}(1-D)(1-\tilde{\lambda})(\bullet \bullet)
$$

$\bullet \bullet \rightarrow \infty \bullet:$

$$
R_{5}=\frac{2 d-1}{2 d} \frac{(\bullet \bullet)}{(\bullet)}[2(1-D)(1-\tilde{\lambda})(\bullet \bullet)+D(\bullet \bullet)]
$$

The equations of motion for the pair probabilities are then

$$
\frac{d}{d t}(\text { ○ })=2 R_{2}+R_{4}-2 R_{1}
$$




$$
\frac{d}{d t}(\circ \bullet)=R_{1}+R_{5}-R_{2}-R_{3}
$$

and

$$
\frac{d}{d t}(\bullet \bullet)=2 R_{3}-R_{4}-2 R_{5}
$$

The active stationary solution of the above equations is

$$
\bar{\rho}=\frac{\lambda[(4 d-3+D) \lambda-2(1-2 d D)]}{(4 d-3+D) \lambda^{2}+2[2 d(D+2)-3] \lambda+4(2 d-1) D},
$$

and

$$
\overline{(\bullet \bullet)}=\frac{\lambda}{\lambda+2} \bar{\rho}
$$

For $\lambda<2(1-2 d D) /(4 d-3+D)$, only the trivial solution $(\bar{\rho}=0)$ exists. If $D \geq D^{*}=1 / 2 d$, however, the active solution exists for any $\lambda>0$. The phase transition occurs at

$$
\lambda_{c}=\left\{\begin{aligned}
\frac{2(1-2 d D)}{4 d-3+D}, & D<D^{*}=\frac{1}{2 d} \\
0, & D>D^{*}
\end{aligned}\right.
$$

Thus the pair approximation predicts a nonzero critical creation rate only for diffusion probabilities $D<D^{*}=1 /(2 d)$; for larger values of $D$, there is a nonzero particle density for any $\lambda>0$, as in the one-site approximation. For $D=0$, we have $\lambda_{c}=2,2 / 5$, and $2 / 9$ in one, two and three dimensions, respectively; the corresponding values from simulation are $\lambda_{c}=5.368(1), 1.0156(1)$, and $0.475(1)$. [We note that the pair approximation results derived above differ slightly from those given in [2] since in the latter case the annihilation rate for a $\mathrm{NN}$ particle pair is taken as $(1-D) /(1+\lambda)$, i.e., $d$ times the rate given in Eq. (11).]

Katori and Konno [7] proved that the prediction of $D^{*}<1$, furnished by the pair approximation, is wrong for $d \leq 2$. That is, in one and two dimensions, $\lambda_{c}>0$ for any $D<1$. In the following section we investigate how $\lambda_{c}$ tends to zero as $D \rightarrow 1$ in one and two dimensions, and determine $D^{*}$ in the three-dimensional case. 


\section{SIMULATIONS}

We use Monte Carlo simulations to obtain accurate values of the critical creation rate $\lambda_{c}(D)$ and the critical exponents of the PAM in one, two, and three dimensions.

\section{A. One dimension}

\section{Spreading behavior}

A well established method for determining the critical point and certain critical exponents is through the study of propagation of activity, starting from a localized seed, as proposed

long ago by Grassberger and de la Torre [16]. One studies the activity in a large set of trials, all starting from a configuration very close to the absorbing state. Here the initial configuration is that of a single pair of particles at the two central sites, in an otherwise empty lattice. Each trial ends when it reaches the absorbing state, or at a maximum time, $t_{\text {max }}$, chosen such that the activity never reaches the edges of the system (in any trial) for $t \leq t_{\max }$.

For $\lambda>\lambda_{c}$ there is a nonzero probability that the process survives as $t \rightarrow \infty$; for $\lambda \leq \lambda_{c}$ the process dies with probability 1 . Of primary interest are $P(t)$, the probability of surviving until time $t$ or greater, $n(t)$, the mean number of particles (averaged over all trials), and $R^{2}(t)$, the mean-square distance of particles from the origin. At the critical point these quantities follow asymptotic power laws,

$$
\begin{aligned}
P(t) & \propto t^{-\delta} \\
n(t) & \propto t^{\eta} \\
R^{2}(t) & \propto t^{z_{s p}}
\end{aligned}
$$

The exponents $\delta, \eta$, and $z_{s p}$ satisfy the hyperscaling relation $4 \delta+2 \eta=d z_{s p}$, in $d \leq 4$ dimensions [16]. (We note that $z_{s p}$ is related to the usual dynamic exponent $z$ via $z_{s p}=2 / z$.)

We study activity spreading in one dimension using samples of from $10^{6}$ or $2 \times 10^{6}$ trials for each $\lambda$ value of interest. The maximum time $t_{\max }=15000$ for $D \leq 0.7,30000$ for $D=0.8$ and 0.9 , and 60000 for $D=0.95$. (As $D$ increases, the asymptotic power-law behavior occurs at ever later times.) To ensure that activity never reaches the borders, we use a lattice size of $L=50000$ for $t_{\max }=15000$, and $L=80000$ for the longer studies. 
A study performed at a given value of $\lambda$ is used to generate results for nearby values using sample reweighting [17].

To locate the critical point, we use the criterion of power-law behavior of $n(t)$; Fig. 2 illustrates the analysis for $D=0.3$. The main graph is a log-log plot of $n(t)$ showing an apparent power law for $\lambda=3.4687$. The curves for nearby values (specifically, $\lambda=3.4681$, 3.4684, 3.4690, and 3.4693, obtained via reweighting), cannot be distinguished on the scale of this graph, but if we plot $n^{*} \equiv n / t^{\eta}$, the curves for different $\lambda$ values fan out (upper inset), with upward curvature indicating a supercritical value of $\lambda$ and vice-versa.

The exponent $\eta$ is estimated via analysis of the local slope, $\eta(t)$, defined as the inclination of a least-square linear fit to the data (on logarithmic scales), on the interval $[t / a$, at]. (The choice of the factor a represents a compromise between high resolution, for smaller $a$, and insensitivity to fluctuations, for larger values; here we use $a=2.59$.) Plotting $\eta(t)$ versus $1 / t$ (lower inset of Fig. 21) allows one to estimate $\lambda_{c}$ (the curves for $\lambda>\lambda_{c}$ veer upward, and vice-versa), and to estimate the critical exponent $\eta$ by extrapolating $\eta(t)$ to $1 / t \rightarrow 0$. The main source of uncertainty in the exponent estimates is the uncertainty in $\lambda_{c}$ itself. An analogous procedure is used to estimate exponents $\delta$ and $z_{s p}$. In Table I we list the critical parameters and spreading exponents found via spreading simulations combined with local-slopes analysis.

For $D=0$, the critical parameter for the PAM, $\lambda_{c}(0)=5.368(1)$, is considerably larger than that of the contact process $\left(\lambda_{c}=3.29785(2)\right)$, as expected since here each annihilation event removes two particles. (The fact that $\lambda_{c}$ is less than twice the corresponding value in the CP may be attributed to the tendency for particles to cluster: removing two particles may eliminate additional pairs, thus reducing the effective rate of annihilation.)

For all diffusion probabilities studied, our estimates for the critical exponents are in good accord with the DP values $\delta=0.15947(3), \eta=0.31368(4)$, and $z=1.26523(3)$ [2]. A plot of the phase boundary in the $\lambda$-D plane (see Fig. 3) suggests that $\lambda_{c} \rightarrow 0$ as $D \rightarrow 1$, so that $D^{*}=1$ in agreement with the Katori-Konno theorem. Extrapolation of $D$ versus $\lambda_{c}$, using a fourth-order polynomial fit to the data for $D \geq 0.6$, yields $D=1.0005$ for $\lambda_{c}=0$, confirming to high precision that $\lambda_{c}>0$ for $D<1$. 


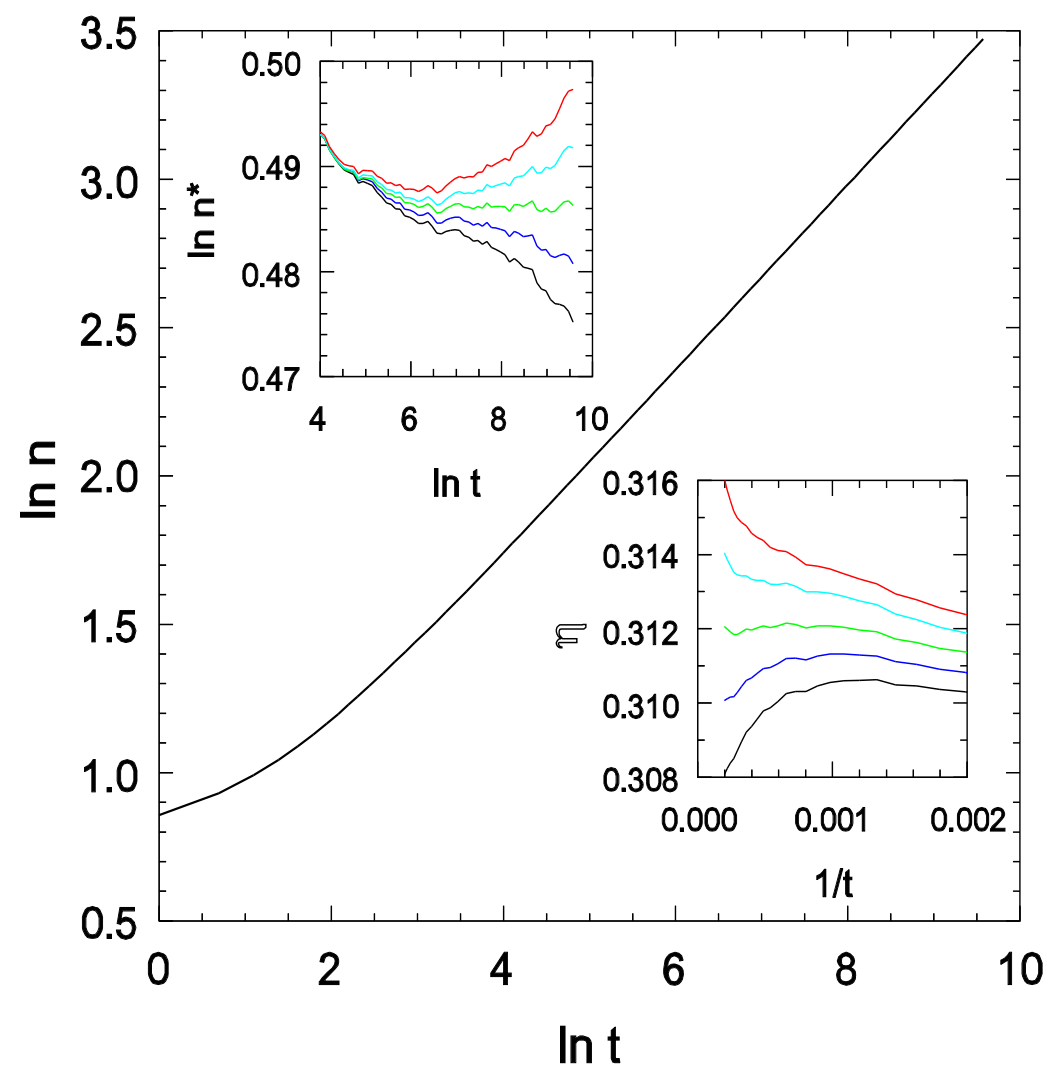

FIG. 2: Main graph: $n(t)$ on log scales for the one-dimensional PAM with $D=0.3$ and $\lambda=3.4687$. Upper inset: $n^{*}=n / t^{\eta}$ on log scales, for (lower to upper) $\lambda=3.4681,3.4684,3.4687,3.4690$, and 3.4693. Lower inset: local slopes $\eta(t)$ for the same set of $\lambda$ values.

\section{B. Two dimensions}

\section{Steady-state behavior}

We encounter rather large uncertainties in studies of spreading behavior of the twodimensional PAM, and so turn to the steady-state approach to investigate this system (on the square lattice). In these studies we initialize the system with all sites occupied, and allow it to evolve until it attains a quasistationary (QS) regime, in which bulk properties such as the particle density $\rho$, averaged over surviving realizations, are time-independent. According to the finite-size scaling hypothesis [18, 19], the QS properties depend on system size $L$ through the ratio $L / \xi$, or equivalently through the scaling variable $\Delta L^{1 / \nu_{\perp}}$, where 


\begin{tabular}{|c|c|c|c|c|}
\hline$D$ & $\lambda_{c}$ & $\delta$ & $\eta$ & $z_{s p}$ \\
\hline \hline 0.0 & $5.3720(5)$ & $0.159(1)$ & $0.315(1)$ & $1.266(2)$ \\
0.1 & $4.6709(2)$ & $0.161(1)$ & $0.314(2)$ & $1.264(1)$ \\
0.2 & $4.0417(2)$ & $0.160(1)$ & $0.314(1)$ & $1.266(1)$ \\
0.3 & $3.4687(2)$ & $0.162(1)$ & $0.312(1)$ & $1.268(2)$ \\
0.4 & $2.9411(2)$ & $0.160(2)$ & $0.314(2)$ & $1.264(2)$ \\
0.5 & $2.4473(1)$ & $0.159(1)$ & $0.315(2)$ & $1.267(3)$ \\
0.6 & $1.9778(2)$ & $0.159(1)$ & $0.315(2)$ & $1.266(1)$ \\
0.7 & $1.5231(2)$ & $0.158(2)$ & $0.315(1)$ & $1.265(2)$ \\
0.8 & $1.0684(2)$ & $0.159(2)$ & $0.315(2)$ & $1.265(2)$ \\
0.9 & $0.5891(1)$ & $0.161(1)$ & $0.315(1)$ & $1.267(3)$ \\
0.95 & $0.3214(1)$ & $0.159(2)$ & $0.318(3)$ & $1.266(4)$ \\
\hline
\end{tabular}

TABLE I: Results of spreading simulations for the PAM in one dimension.

$\Delta \equiv \lambda-\lambda_{c}$. Expressing the order parameter as a function of $\Delta$ and $L$, we have

$$
\rho(\Delta, L) \propto L^{-\beta / \nu_{\perp}} f\left(\Delta L^{1 / \nu_{\perp}}\right)
$$

with $f(x) \propto x^{\beta}$ as $x \rightarrow \infty$. At the critical point, $\Delta=0$,

$$
\rho(0, L) \propto L^{-\beta / \nu_{\perp}}
$$

Thus an asymptotic power-law dependence of $\rho$ on $L$ is a useful criterion for criticality.

We study the QS density as a function of system size to locate the critical point, using sizes $L=25,50,100, \ldots, 800$. The relaxation time varies from $\tau=800$ for the smallest size, to $\tau=200000$ for the largest; the number of realizations varies from 500 to 10000 . Using the power-law criterion, we obtain the estimates for $\lambda_{c}$ listed in Table II. It is worth mentioning that the values for $\lambda_{c}$, for $D=0$ and $D=0.1$ are in good agreement with those obtained in the preliminary spreading behavior studies.

In Fig. 4 we verify the scaling collapse of the order parameter, plotting $x \equiv L^{\beta / \nu_{\perp}} \rho$ versus $y \equiv \Delta L^{1 / \nu_{\perp}}$, for system sizes $L=16,32,64,128$, and 256. A good collapse is obtained using the DP values $\nu_{\perp}=0.733$ and $\beta / \nu_{\perp}=0.795[2]$. The data are consistent with the scaling law $\rho \propto \Delta^{\beta}$, using the $\mathrm{DP}$ value $\beta=0.583(4)[2]$. 


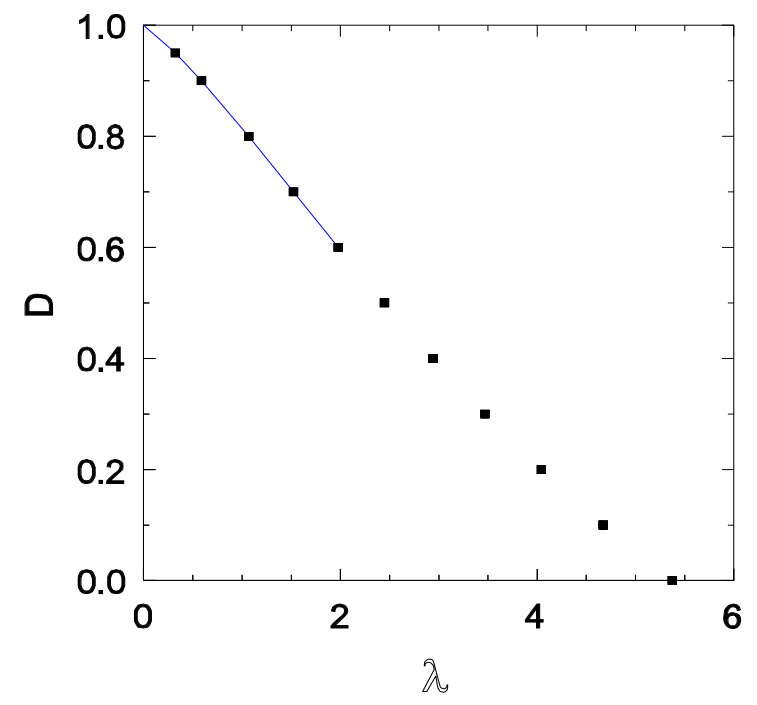

FIG. 3: Points along the critical line in the $\lambda-D$ plane in one dimension, as determined via simulation. Error bars are smaller than symbols. The solid line is a quartic fit to the six points with largest $D$.

\begin{tabular}{|c|c|}
\hline$D$ & $\lambda_{c}$ \\
\hline \hline 0.0 & $1.0156(1)$ \\
0.1 & $0.7877(1)$ \\
0.2 & $0.5890(5)$ \\
0.3 & $0.4166(1)$ \\
0.4 & $0.2685(5)$ \\
0.5 & $0.1462(2)$ \\
0.6 & $0.056(1)$ \\
\hline
\end{tabular}

TABLE II: Critical parameters obtained through steady-state simulations in two dimensions.

\section{Quasistationary simulations}

As $D$ approaches 0.7 the critical value $\lambda_{c}$ becomes very small. We require an efficient simulation method to obtain precise estimates for the critical value for larger diffusion rates, in particular, to determine how $\lambda_{c}$ tends to zero as $D$ increases. For this purpose we use quasistationary (QS) simulations, which sample directly the QS probability distribution, that is, the long-time distribution conditioned on survival. The details of the method are 


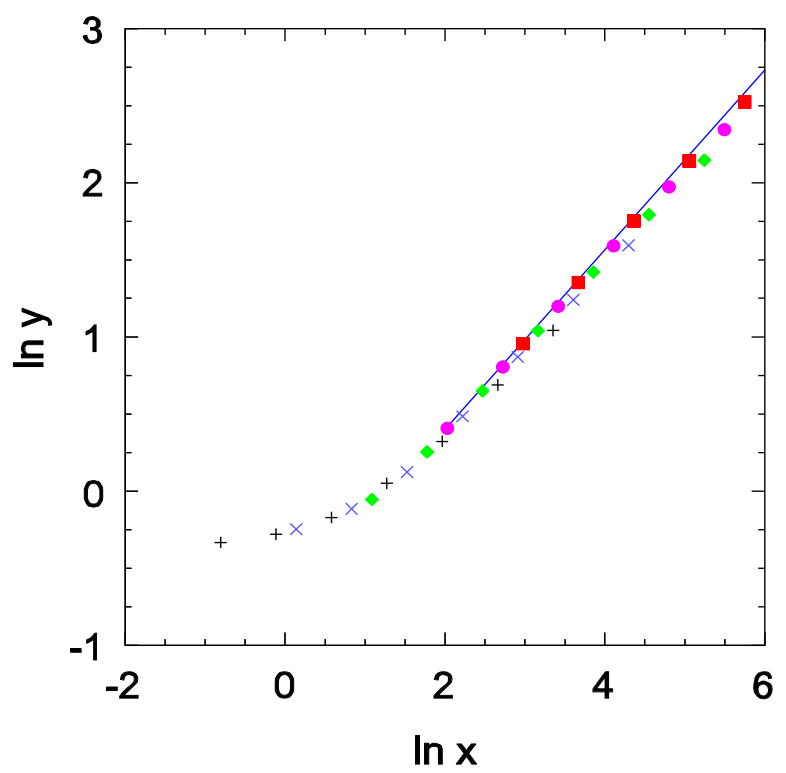

FIG. 4: (Color online) Scaling plot of the stationary density in the two-dimensional PAM with $D=0$. System sizes $L=16(+) ; 32(\times) ; 64$ (diamonds); $128(\bullet)$ and 256 (squares). The slope of the straight line is 0.583 .

explained in Ref. [20]. To obtain these results we use lattice sizes $L=100,200,400$ and 800 for $D=0.7$, and include studies of larger systems for higher diffusion rates (up to $L=6400$, for $D \geq 0.78)$. The critical point is determined via the criteria of power-law scaling of the density and mean lifetime with system size, and convergence of the moment ratio $m=\left\langle\rho^{2}\right\rangle / \rho^{2}$ to a finite limiting value as $L \rightarrow \infty$, as discussed in [21]. (The lifetime $\tau$ is expected to follow $\tau \sim L^{z}$.) Using this method we obtain the values listed in Table III. We note that our results for $\beta / \nu_{\perp}, z$, and the limiting moment ratio $m_{c}$ are consistent with the known DP values of $0.795(10), 1.7674(6)$, and 1.3257(5), respectively [2, 17, 21].

For $D=0.8, \lambda_{c}$ is of order $10^{-4}$, and a precise determination becomes very difficult due to the small number of particles present in the system. Reliable determination of $\lambda_{c}$ for larger diffusion rates would therefore require studies of even larger systems, which was deemed impractical.

We find that $\lambda_{c}(D)$ can be fit quite well using an expression of the form 


\begin{tabular}{|c|c|}
\hline$D$ & $\lambda_{c}$ \\
\hline \hline 0.60 & $0.05632(3)$ \\
0.70 & $0.00940(5)$ \\
0.73 & $0.003957(3)$ \\
0.78 & $0.0004815(7)$ \\
0.80 & $0.00015(2)$ \\
\hline
\end{tabular}

TABLE III: Critical parameters obtained through quasistationary simulations in two dimensions.

$$
\lambda_{c}=A \exp \left[-\frac{C}{(1-D)^{\gamma}}\right] .
$$

Applied to the data for $D \geq 0.4$, a least-squares procedure yields $\gamma=1.41(2), C=0.984(2)$, and $A=2.02(2)$. The good quality of the fit is evident in the inset of Fig. 5. Thus, while a plot of the data on linear scale might suggest that $\lambda_{c} \rightarrow 0$ at some diffusion rate between 0.8 and 1 (see Fig. 5, main graph), our results are in fact consistent with $\lambda_{c}$ nonzero, though very small, for diffusion rates between 0.7 and unity.

\section{Three dimensions}

We employed quasistationary simulations to determine $\lambda_{c}(D)$ for the PAM on the simple cubic lattice. For relatively small diffusion rates good results are obtained using lattice sizes $L=8,16,24,36$, and 54 . For diffusion rates greater than about 0.25 , however, there are substantial finite-size effects, and to observe clear signs of DP-like scaling we need to study larger systems ( $L=80$ and 120 in addition to the sizes mentioned above). The results (see TableIV]and Fig. [6), show that in this case $\lambda_{c}$ does fall to zero at a diffusion rate considerably less than unity; extrapolation of the data to $\lambda=0$ yields $D^{*}=0.333(3)$. The critical exponents determined via finite-size scaling analysis, $\beta / \nu_{\perp}=1.40(1)$ and $z=1.94(2)$, are once again in good agreement with literature values of 1.39(3) and 1.919(4), respectively. Our study yields the moment ratio value $m=1.47(1)$ for the three-dimensional models in the DP universality class; to our knowledge this quantity has not been determined previously. For $D>D^{*}$, the particle density is expected to tend to zero linearly with $\lambda$, as the reproduction rate approaches zero. We have verified this behavior (down to $\lambda=10^{-4}$ ) for $D=0.8$. 


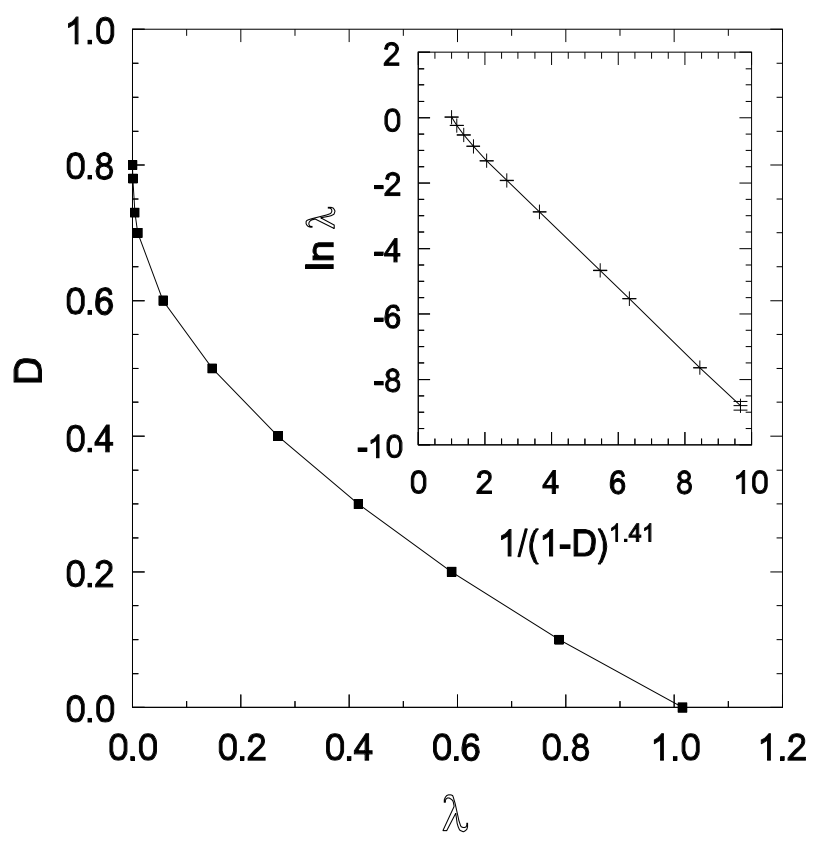

FIG. 5: Critical line of the two-dimensional PAM. Inset: the same data plotted as $\ln \lambda_{c}$ versus $1 /(1-D)^{1.41}$.

\begin{tabular}{|c|c|}
\hline$D$ & $\lambda_{c}$ \\
\hline \hline 0.0 & $0.47390(5)$ \\
0.1 & $0.2943(1)$ \\
0.2 & $0.1420(1)$ \\
0.25 & $0.07790(5)$ \\
0.28 & $0.04487(3)$ \\
0.31 & $0.01762(2)$ \\
0.32 & $0.0103(1)$ \\
\hline
\end{tabular}

TABLE IV: Critical parameters obtained through quasistationary simulations in three dimensions.

\section{DISCUSSION}

We study the phase boundary of the pair annihilation model in the reproduction rate diffusion probability $(\lambda-D)$ plane. Our simulation results are consistent with the theorem 


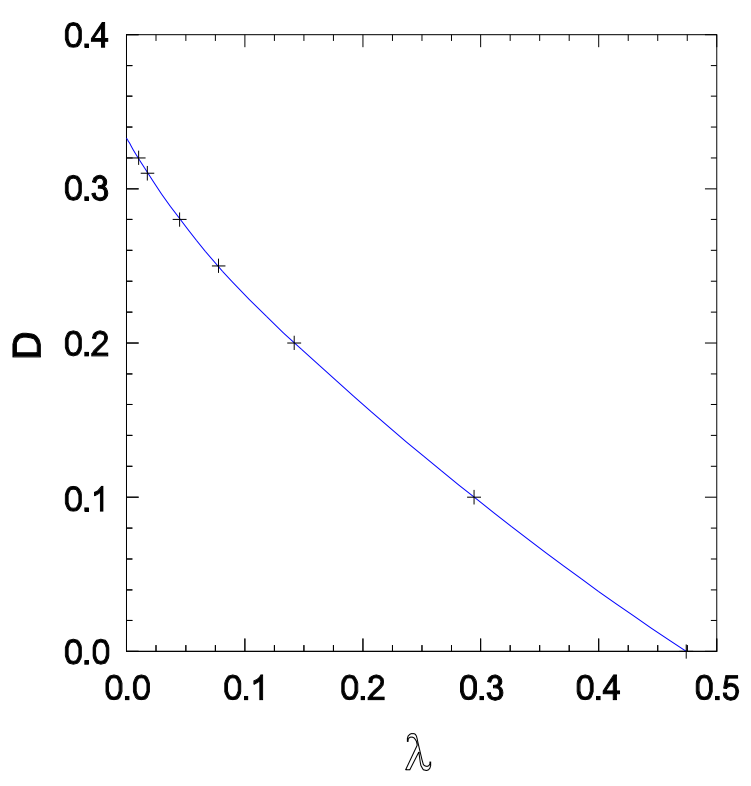

FIG. 6: Critical line of the PAM in three dimensions; error bars are smaller than symbols. The solid line is a cubic fit to the data, yielding $D^{*}=0.333(3)$.

proven some time ago by Katori and Konno [7], namely that in one and two dimensions, $\lambda_{c}>0$ for any $D<1$. The pair approximation is in conflict with this result, as it predicts that in any number of dimensions, there is a diffusion probability $D^{*}<1$, above which $\lambda_{c}=0$. In one dimension the behavior (in simulations) is straightforward, as $\lambda_{c} \propto 1-D$ for $D \simeq 1$. In two dimensions however it is quite subtle, as $\lambda_{c}$ becomes exponentially small as $D \rightarrow 1$, and a cursory analysis could well give the impression that $\lambda_{c}$ is actually zero at some value of $D$ between 0.8 and unity. Finally in three dimensions the pair approximation prediction is verified qualitatively; we find $D^{*}=0.333(3)$ in this case, while the PA yields $D^{*}=1 / 6$. Intuitively, the unusual behavior of $\lambda_{c}(D)$ in two dimensions can be understood as a consequence of $d=2$ marking a critical dimension for the recurrence of a random walk. Our simulation results for critical exponents and the moment ratio $m$ are consistent with the directed percolation values, as expected. Given the qualitative failure of the pair approximation in one and two dimensions, it is natural to ask whether approximations using larger clusters would predict the phase diagram correctly. This strikes us as unlikely, since 
cluster approximations have been found to be insensitive to subtle effects involving diffusion and/or multiparticle rules in other cases [15, 22, 23].

Acknowlegdments This work was supported by CNPq, Brazil.

[1] T. E. Harris, Ann. Probab. 2, 969 (1974).

[2] J. Marro, and R. Dickman, Nonequilibrium Phase Transitions in Lattice Models (Cambridge University Press, Cambridge, 1999).

[3] M. Henkel, H. Hinrichsen, and S. Lübeck, Nonequilibrium phase transitions (Springer-Verlag, Berlin, 2008).

[4] G. Ódor, Universality in Nonequilibrium Lattice Systems (World Scientific, Singapore, 2008).

[5] R. Dickman, Phys. Rev. B40, 7005 (1989).

[6] R. Dickman, Phys. Rev. A42, 6985 (1990).

[7] K. Katori and N. Konno, Physica A 186, 578 (1992).

[8] M. Bramson and L. Gray, Z. Wahrsch. Verw. Gebiete 68, 447 (1985).

[9] H. K. Janssen, Z. Phys. B 42, 151 (1981).

[10] P. Grassberger, Z. Phys. B 47, 365 (1982).

[11] D. C. Torney and H. E. McConnell, Proc. Roy. Soc., Lond. A387, 147 (1983).

[12] D. ben-Avraham and S. Havlin, Diffusion and Reactions in Fractals and Disordered Systems (Cambridge University Press, Cambridge, 2000.)

[13] P. J. Flory, J. Am. Chem. Soc. 61, 1518 (1939); B. Widom, J. Chem. Phys. 44, 3888 (1966); 584043 (1973).

[14] M. J. de Oliveira, T. Tomé, and R. Dickman, Phys. Rev A46, 6294 (1992).

[15] D. ben-Avraham and J. Köhler (1992). Phys. Rev. A 45, 8358.

[16] Grassberger P., and de la Torre, A. (1979). Ann. Phys. (N. Y.) 122, 373.

[17] R. Dickman, Phys. Rev. E60, R2441 (1999).

[18] M. E. Fisher. Proceedings of the Enrico Fermi International School of Physics, Vol. 51, edited by M.S. Green (Academic Press, Varenna, Italy, 1971). M. E. Fisher and M. N. Barber, Phys. Rev. Lett. 28, 1516 (1972).

[19] M. N. Barber, in Phase Transitions and Critical Phenomena, Vol. 8, edited by C. Domb and 
J. L. Lebowitz, (Academic Press, New York, 1983).

[20] M. M. de Oliveira and R. Dickman, Phys. Rev. E 71, 016129 (2005).

[21] R. Dickman and J. K. Leal da Silva, Phys. Rev. E, 58, 4266 (1998).

[22] A. A. Ferreira and J. F. Fontanari, J. Phys. A42, 085004 (2009).

[23] G. Ódor and R. Dickman, J. Stat. Mech. (2009) P08024. 